\title{
New Magnetic Translation/Rotation Drive by Use of Magnetic Particles with Specific Gravity Smaller than a Liquid
}

\author{
Makoto Nokata
}

Additional information is available at the end of the chapter

http://dx.doi.org/10.5772/50999

\section{Introduction}

Magnetic fluid has unique properties[1], by which it can, not only be attracted to, but also solidified by magnets. Many researchers have proposed useful applications for these properties, such as in dampers, sensors, actuators [2][3] and medical applications[4]-[6]. J.Furusho et al. [7] developed MR-Fluid Actuator, controlled it by use of torque Feedback. He also developed 6-DOF Rehabilitation System driven by ER-Actuator[8]. The functional fluid has performed well in clinical use. However, as all Magnetic fluids have a greater specific gravity than liquids like water, the applications are restricted.

This chapter presents a new type of magnetic fluid and the rotational drive with simple magnetic field. This chapter consists of two sections.

The first section describes the development of a unique magnetic fluid and the proposal of a driving principle by use of the magnetic fluid. We have developed a lighter fluid than liquid, mixed it with liquid, and created a new impetus in this research. We have proposed a driving principle and developed the verification models and the generator of magnetic field. It became clear that our proposed driving principle results from collision of the magnetic particles to the outer cover, deformation of the whole form of magnetic particles and movement of the center of gravity.

The second section describes an advanced translation drive that produces non-slipping motion in soft and closed environment by use of the magnetic fluid. New movement principle of the robot, which has a soft and deformable body that can move through a confined space is proposed. The mechanism of a toy water snake is applied to this principle. Magnetic particles inside the water balloon are affected by the external magnetic field and push the inner side of a balloon to the direction of a magnetic field. We construct an experimental model to verify the proposed principle, the sliding movement is measured using the model. 


\section{A unique magnetic fluid and driving principle}

This section describes the development of a unique magnetic fluid and the proposal of a driving principle by use of the magnetic fluid.

\subsection{Principle of magnetic drive}

The explanation shows the magnetic drive principle by use of floating magnetic fluid and a simple magnetic field.

Fig.1 shows the schematic diagram of the rolling object that is driven by the proposed magnetic drive principle. The functional section(1) and the liquid(2) are sealed in the interior by the outer cover(3).

The inner wall(4) and outer wall(5) of the outer cover are covered with the small projections or a textured material. The projections play the role of creating friction. Floating magnetic particles(6) are employed in a magnetic field. The specific gravity of these magnetic particles is smaller than the liquid, so they gather in the upper part of the rolling object when no magnetic field is applied.

When a magnetic field is applied, the magnetic particles move in the direction of the magnetic field quickly. The path they take is just inside the outer cover, because both the magnetism and buoyancy are working on the magnetic particles. The particles collide with the projections on the inner wall of the outer cover (Fig. 1(b)).

The external side of the outer cover does not slip on the ground, because there is friction between the ground and the outer wall. Consequently, the rolling object moves in the direction of the magnetic field (Fig. 1(c)). After the magnetic field is removed, the magnetic particles will return to the upper part of the rolling objet slowly (Fig. 1(d)), the rolling object stayed at the same position. By repeating the above, the rolling object can move a long distance.

If the specific gravity of a magnetic particle is larger than the liquid, the object rotates in the opposite direction (Fig.2). The movement becomes unstable because it may move in the opposite direction to the magnetic field.

\subsection{Verification experiment and discussion}

\subsubsection{Float magnetic particle}

In order to realize the above-mentioned principle of operation, magnetic particles with a specific gravity smaller than the liquid are required. Fig. 3 shows a photograph and schematic diagram of the particles. The diameter of each particle is 1-2 $\mathrm{mm}$. The particles are composed of magnetic iron oxide $4.5 \%$, Kerosene $95.2 \%$ and Additives $0.3 \%$.

\subsubsection{Verification models}

Fig.4 shows the photograph of two types of the model used for the verification of the above-mentioned principle. Fig.4(a) is a hard model for multi-directional drive on a solid surface, and Fig.4(b) is a soft model for crawling on uneven ground. 


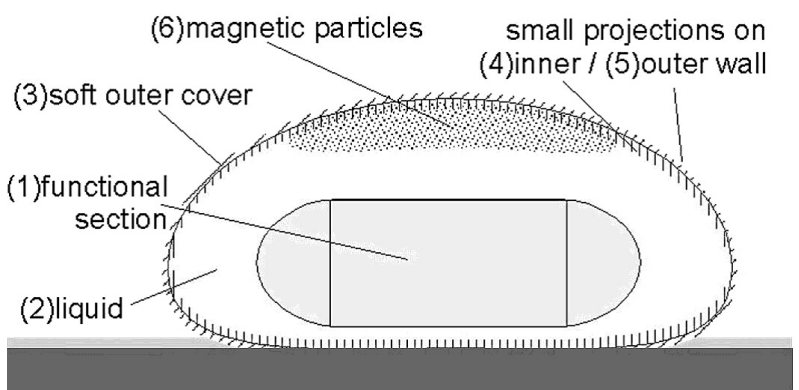

(a) Structure of a rolling object driven by magnetic field

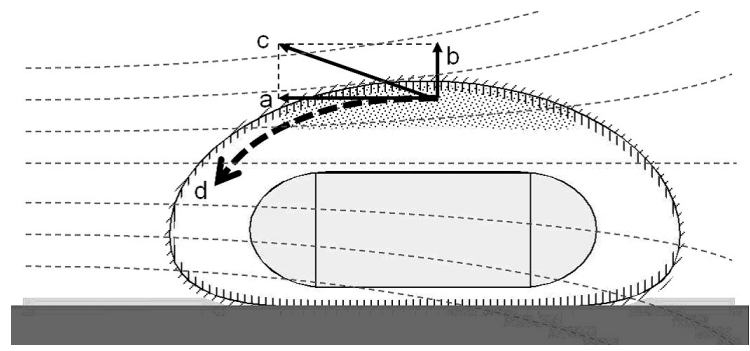

(b) Particles move in the direction of a magnetic field quickly

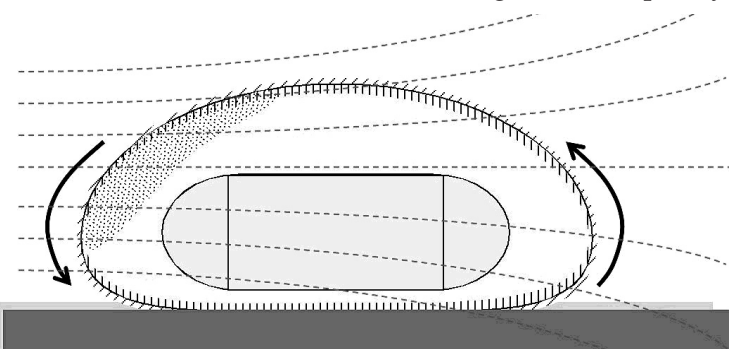

(c) Object rolls and moves

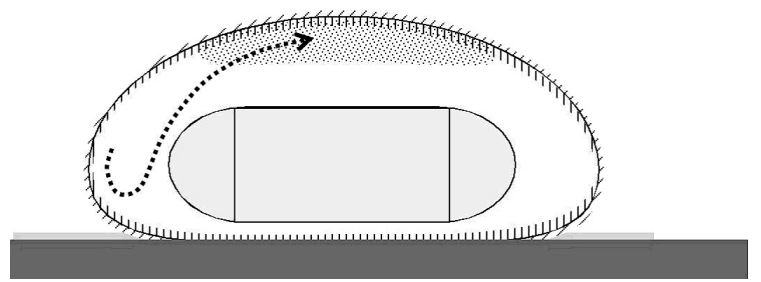

(d) Magnetic particles return to the upper part slowly

Figure 1. Magnetic drive principle with simple magnetic field 


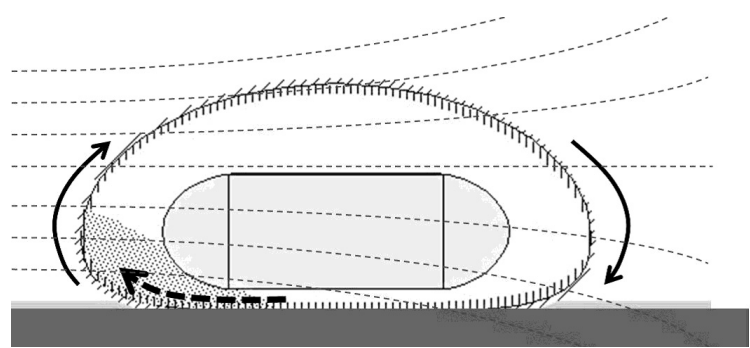

Figure 2. Reverse drive. If the specific gravity of a magnetic particle is larger than a liquid, the rolling object rotates conversely.
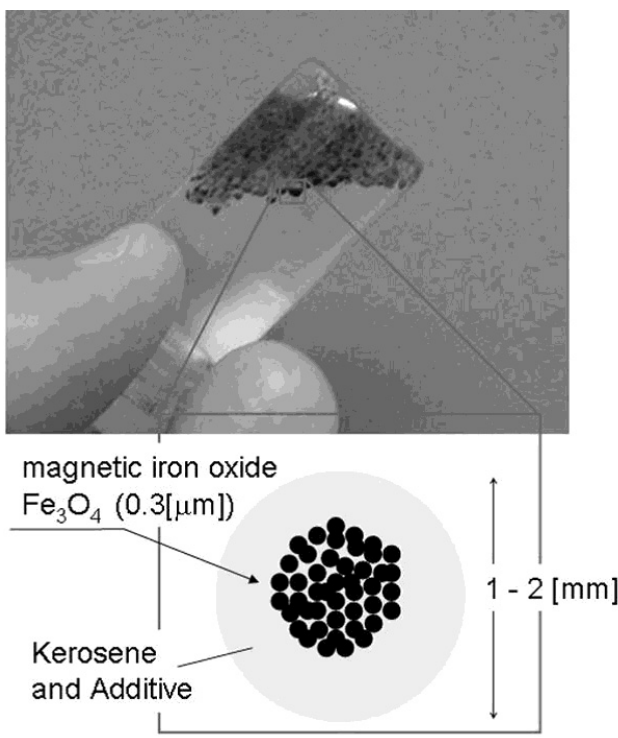

Figure 3. Photograph and schematic diagram of a magnetic particle with specific gravity smaller than a liquid

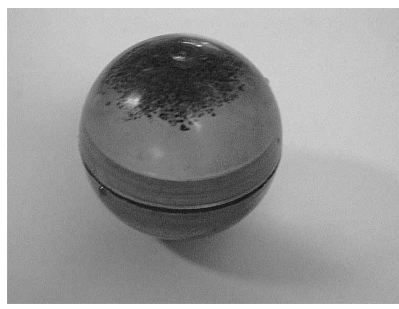

(a)Hard model for omni directional drive

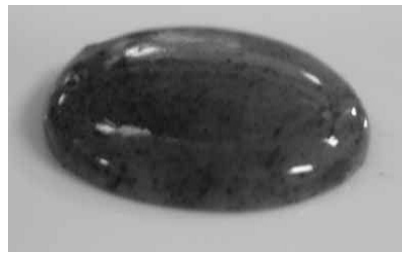

(b)Soft model for crawling on an uneven surface

Figure 4. Two types of the model for the verification of the above-mentioned principle 


\subsubsection{Generator of magnetic field}

Fig. 5 shows the system that generates the magnetic field necessary to move the model, it consists of a solenoid (outer diameter: $100 \mathrm{~mm}$, inner diameter: $50 \mathrm{~mm}$, length: $100 \mathrm{~mm}$, cross-sectional dimension of coil line: $2 \times 5 \mathrm{~mm}, 163$ turns); a power supply (applied voltage: 50 - $900 \mathrm{~V}$, capacitance of capacitor: $10 \mathrm{mF}$, maximum permissible current: $10000 \mathrm{~A}$ ); and a sensor probe that measures the flux density of three axes. The charging time of the power supply is $0-5 \mathrm{~s}$, the solenoid generates a magnetic field with a peak because the stored charge is discharged all at once. Fig.6 shows a graph of the magnetic field generated by the system; about $2 \mathrm{~T}$ can be generated. Fig.7 shows the wave shape of the magnetic flux density at a point on the center axis that is $7 \mathrm{~cm}$ from the edge of the solenoid.

\subsubsection{Results and discussions}

In order to verify the drive principle, an experiment to drive a solid model as shown in Fig.4 (a) was conducted. At first, The magnetic flux density:150 mT was generated horizontally. The model motions captured by camera are shown in Fig.8. The monitor in the captured image shows the value of the flux density. The magnetic particles were shifted to the upper part of the solid model by magnetic force, contacting the inside of the outer cover. All the particles were moved a distance of $2 \mathrm{~cm}$ by one $150 \mathrm{mT}$ magnetic field impulse. As a result, this experiment demonstrated the practicality of the magnetic drive principle using a simple magnetic field. Next, a magnetic flux density of $150 \mathrm{mT}$ was generated obliquely downward. The magnetic particles were shifted obliquely downward and didn't contact the inside the outer cover, so the model didn't move.

Fig.9 and Fig. 11 show the model motions captured by camera, when the magnetic flux density was generated horizontally. Magnetic particles clumped together and adhered to the inside wall. The form of the clump did not disintegrate when shaken and the center of gravity of the clump was maintained (Fig.9(1)). When a magnetic field was applied, the model began to rotate and roll in the direction of rotation, with the clump maintaining its form (Fig.9(2)(3)). When the inclination of the clump in the model became large, each particle began to move

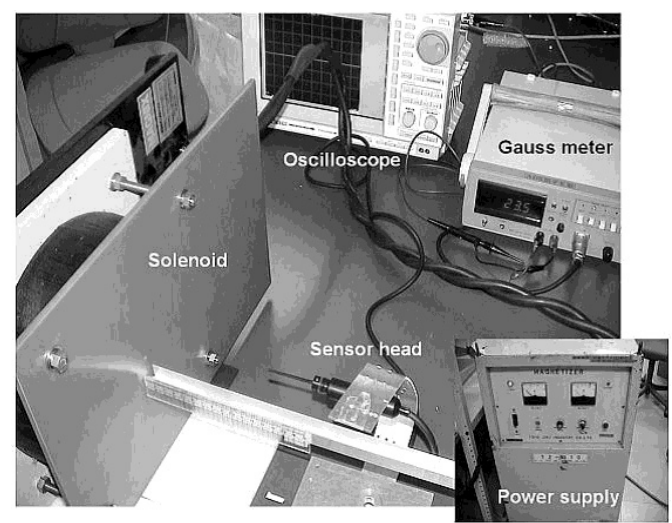

Figure 5. Total system of generating the impulse to the magnetic field for driving the models, it consists of a solenoid, a power supply, and a sensor probe that measures the flux density of three axes. 


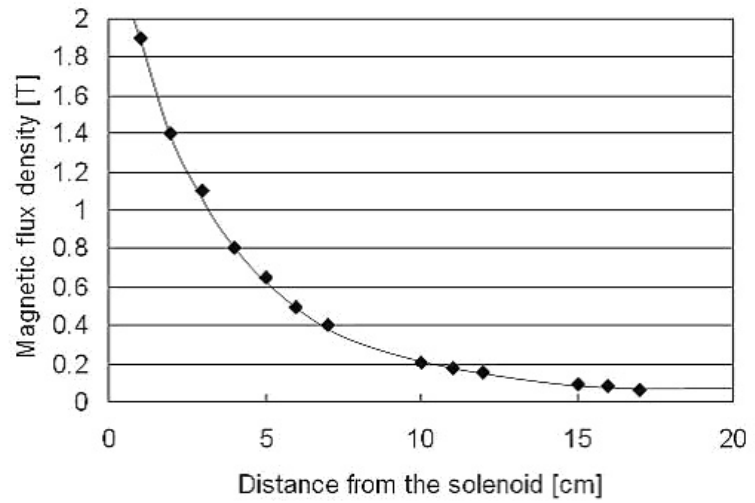

Figure 6. Graph of the magnetic field produced by the system, about 2 Tesla (T) can be generated.

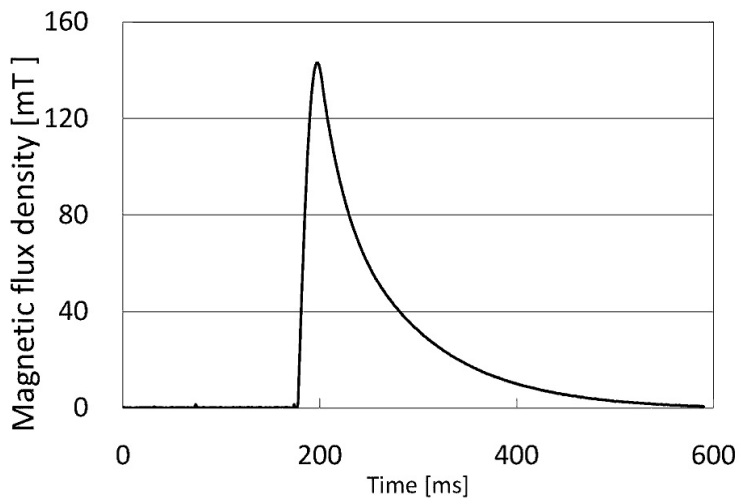

Figure 7. Wave shape of the magnetic flux density at the point of $12 \mathrm{~cm}$ on a center axis from the edge of the solenoid.

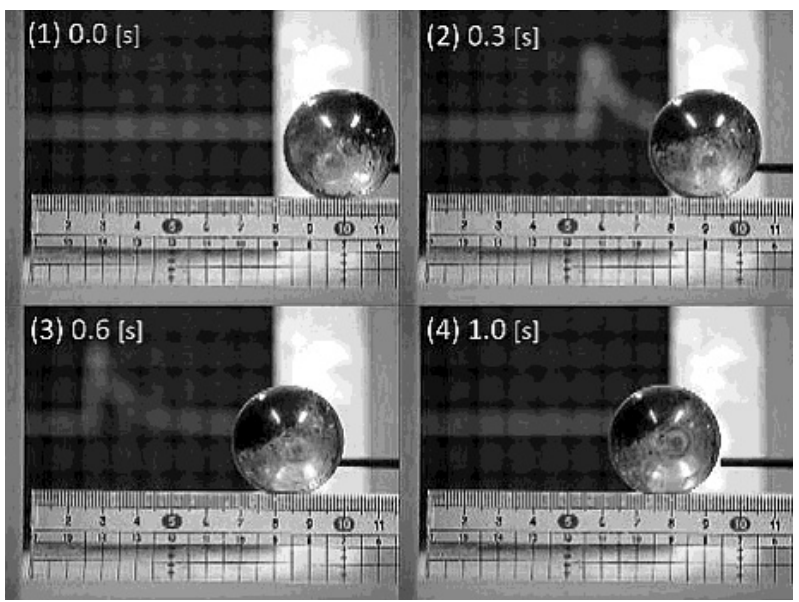

Figure 8. Captured motions of the rolling model. The magnetic flux density:150[mT] was generated horizontally. The monitor in back shows the value of flux density. 


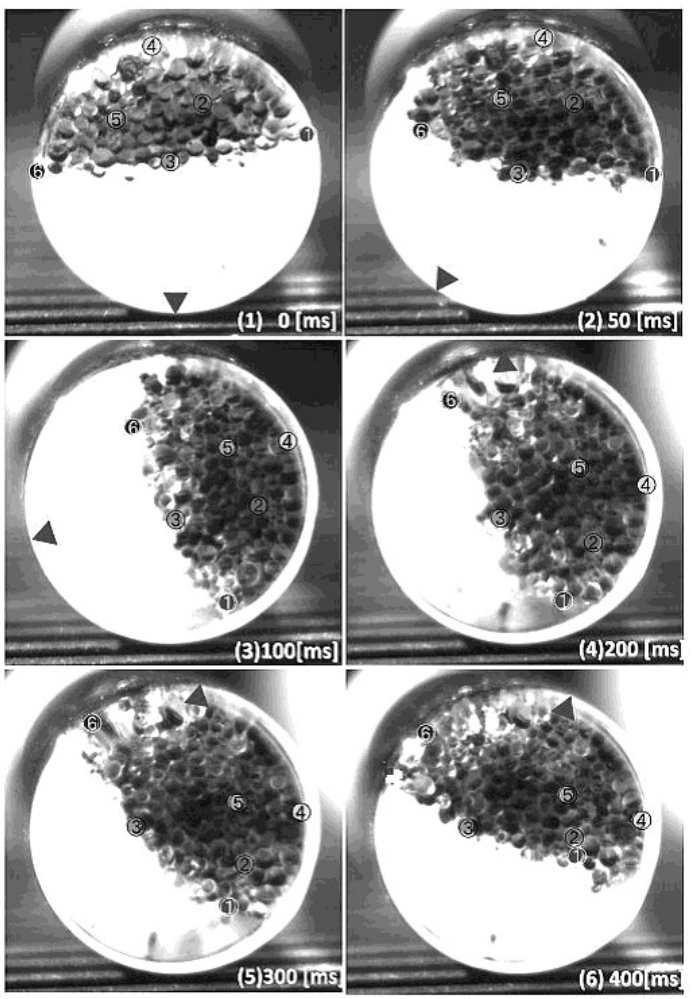

Figure 9. Captured motion which there is slide in the particle

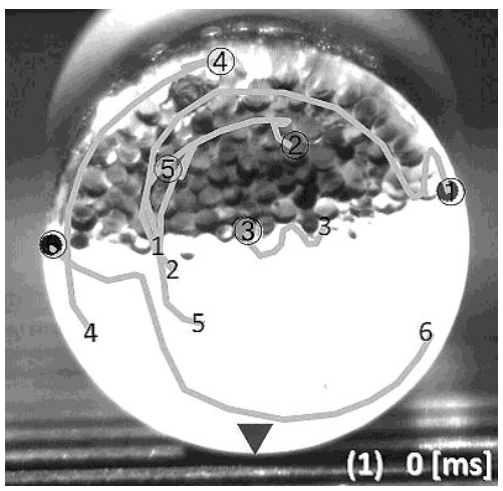

Figure 10. Move Path of each particle 
separately due to its buoyancy, and the shape of the clump changed (Fig.9(4)(5)). The position of the center of gravity became stable, so the rotation stopped (Fig.9(6)). If the form did not change, the model turned back to its original position (Fig.11, left-side figures).

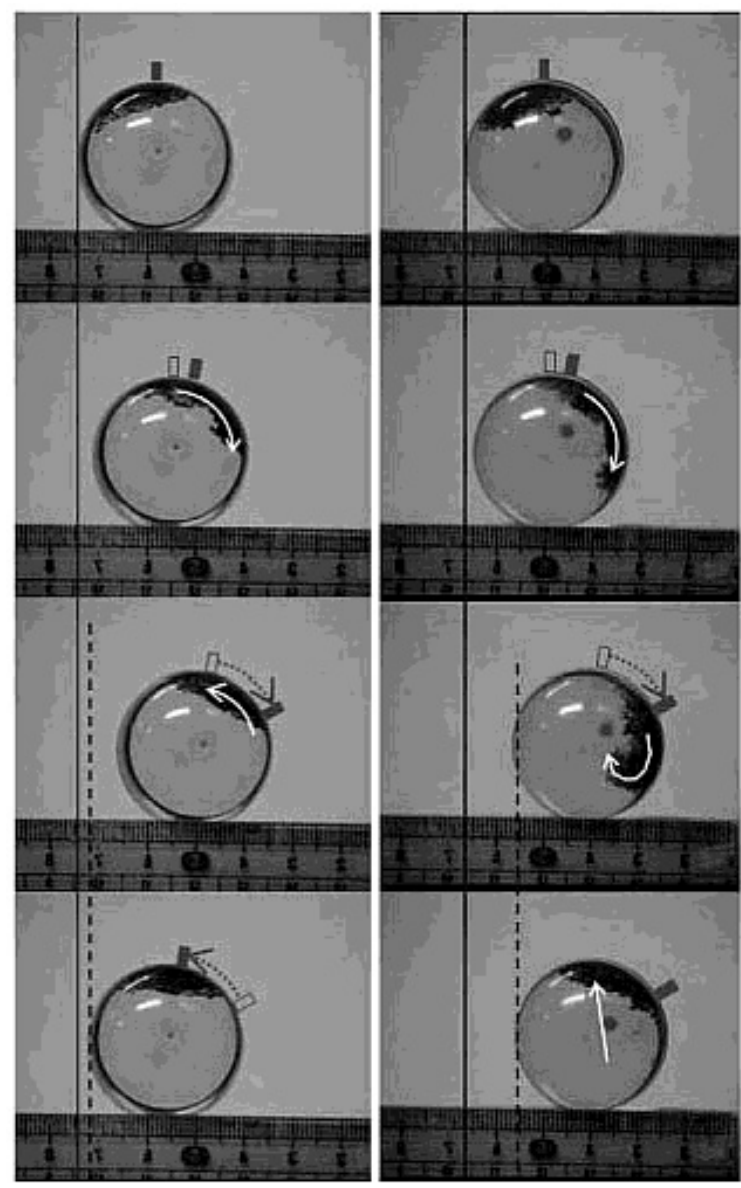

Figure 11. Captured motion which there is slide in the particle and the inner wall.

Fig.10 shows the path of each particle. The particles were circulated by convection, that is, a sequential convection current can cause sequential rolling. This result says that there is another magnetic wave pattern in addition to the impulse one shown in Fig.7. As a result, it became clear that our proposed driving principle results from not only collision of the magnetic particles with the outer cover but also the flow resistance between the particles and the cover. Equation 1 shows the force $F$ produced on the magnetic particles by the external magnetic field.

$$
F=M \frac{d H}{d r}=\frac{[w b m][N / w b]}{[m]}=[N]
$$




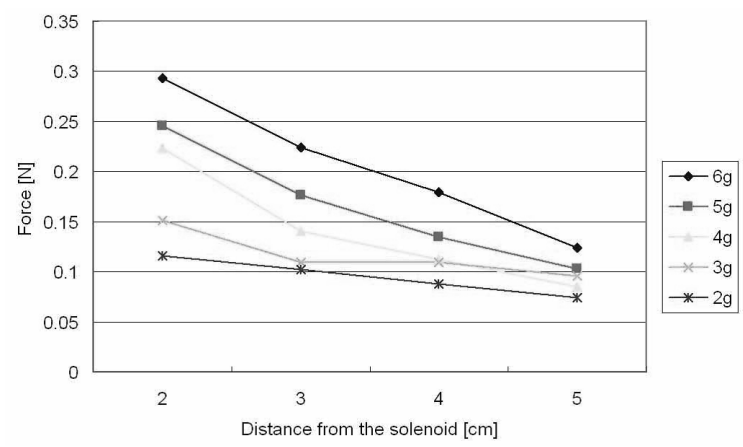

Figure 12. The graph of the measured magnetic field produced by the equipment of Fig.5. The values are measured by the gauss meter for every mass of a magnetic particle.

$M$ is the magnetic moment, $H$ is the magnetic field strength and $r$ is position of the magnetic particle. Fig.12 shows the graph of the measured magnetic field produced by the equipment in Fig.5. The mass, of each magnetic particle is measured using the gauss meter.

\subsection{Applications}

This chapter introduces some mechanisms that make use of the magnetic drive principle.

(1)Ring type (Fig.13(a)) This mechanism consists of an outer cover in ring form and an axis of rotation. The torque can be taken from the main axis.

(2) Propeller type (Fig.13(b)) After a magnetic field is applied and the magnetic particles flow downward, the particles then float back up due to buoyancy. When a particle contacts a propeller, torque is generated.

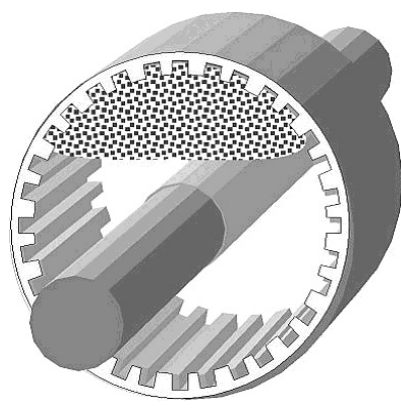

(a) Ring type

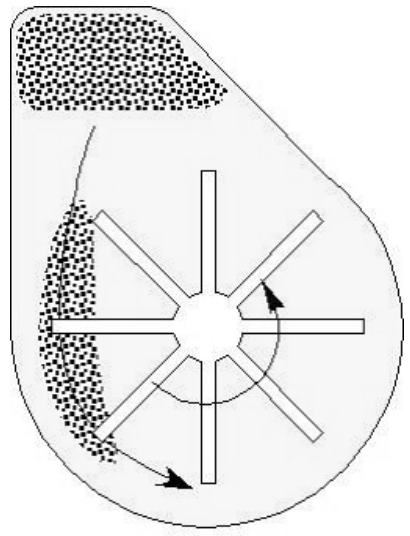

(b) Propeller type

Figure 13. Mechanisms which applied the magnetic drive principle

Fig.14 shows the prototype of Ring type (Fig.13(a)), Fig.15 explains the principle of driving. In order to verify the drive principle, an experiment to drive a simple model was developed. Fig.16 shows the model motions captured by camera, when the magnetic flux density was 

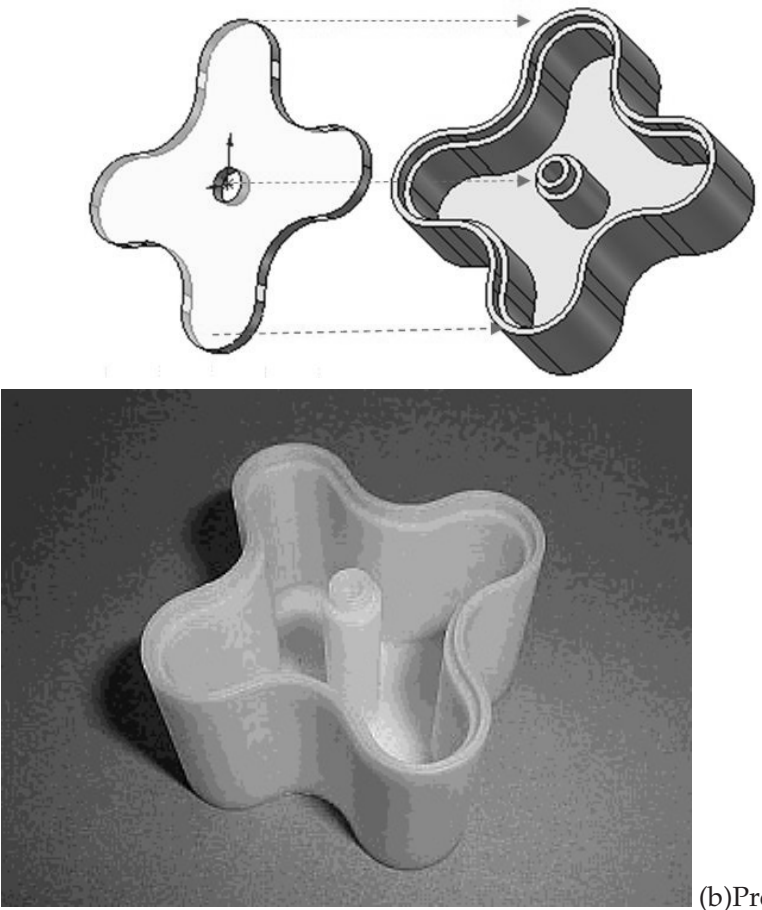

(a)Design of cross wheel

Figure 14. Prototype of ring type (cross wheel)

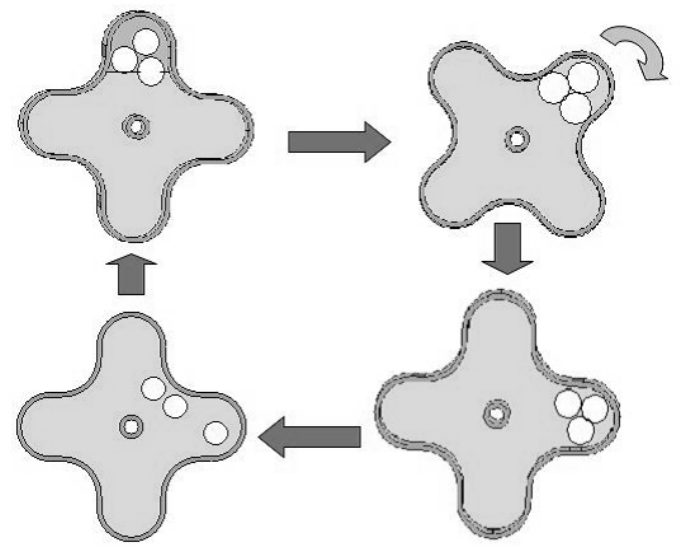

Figure 15. Driving Principle of cross wheel

generated horizontally. Magnetic particles pushed the inside wall of the cross wheel, rotated the wheel, then the model drove itself forward. 
This driving principle could be applied to medical robots shown in Fig.17. One impulse of magnetic force produces 90 degree rotation of cross wheel. This mechanism is able to control the travel distance, little influenced by friction and viscosity of organs[9]. In addition, No actuator and no battery are required for devices inserted in the body. The external magnetic field is suitable for supplying the rotating or driving force to the device. This application has the advantage over a capsule robot that has a permanent magnet mounted in its interior.
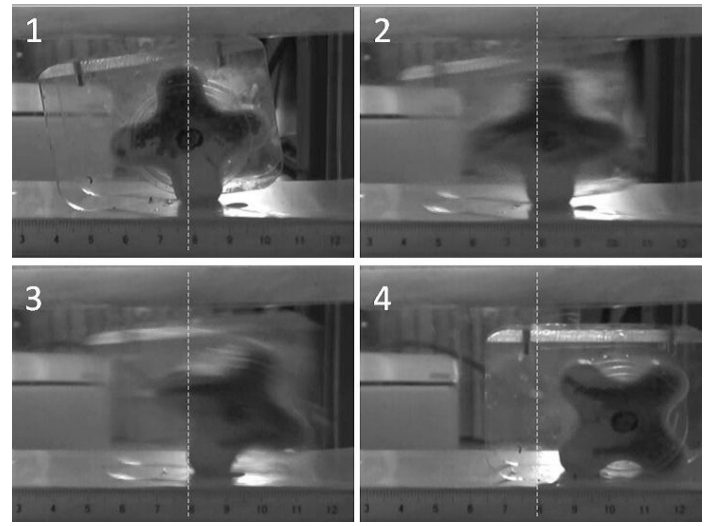

Figure 16. Motions of cross wheel captured by camera

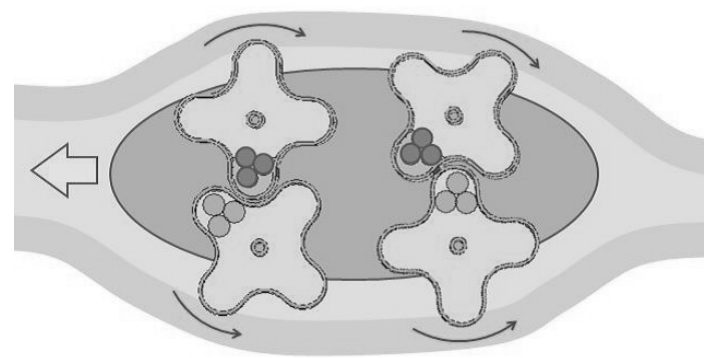

Figure 17. Schematic diagram of internal medical robot in bowel

\section{Deformable robot actuation by magnetic particle}

The endoscope, which is a flexible tube, is used to examine the interior of the human gastrointestinal tract. The standard endoscope is inserted via the mouth or the anus and manually pushed and pulled into the organ to be examined. In contrast, a capsule endoscope is swallowed by a patient and naturally exits the body within eight hours. [10] Another capsule endoscope mounts a permanent magnet inside the body, rotates and moves actively by applying a rotational magnetic field. [11][12] M. Shikanai et al. developed a robotic endoscope that consists of front and rear bodies with bidirectional rotational helical fins. [13] The front and rear bodies are connected by a DC motor. The robot advances through the 
intestines by clockwise rotation of the front body and anticlockwise rotation of the rear body. These capsule-type robots require power supply wires and a permanent magnet. A potential problem with this design is that the rotational drive could cause engulfment. P. Glass et al. developed new controllable capsule endoscopes, the prototypes have used shape memory alloy or micro motors to press elastomer micropillar legs into the gastrointestinal lining to anchor the capsule in place.[14][15] Bio-inspired adhesives by the elastomer micropillar legs to stick the capsule to the intestinal lining allow the clinician to control the progress of the capsule along the patient's gastrointestinal tract. Another type of capsule robot mounts a ferromagnetic material inside the body and is moved by applying a gradient magnetic field. [16] The limitation of this design is that slipping locomotion is affected by the surface friction of organs. A magnetic particle has been reportedly developed that is lighter than liquid, and on mixing with liquid creates a new impetus. [17]

This section presents an advanced locomotion method that produces non-slipping motion in digestive organs and the abdominal cavity by developing an endoscopic robot. The developed endoscopic robot is soft, deformable, and can move through a confined environment of internal organs.

\subsection{Proposal and features of movement principle}

This section describes the proposed movement principle of the robot, which has a soft and deformable body that can move through a confined space. The mechanism of a toy water

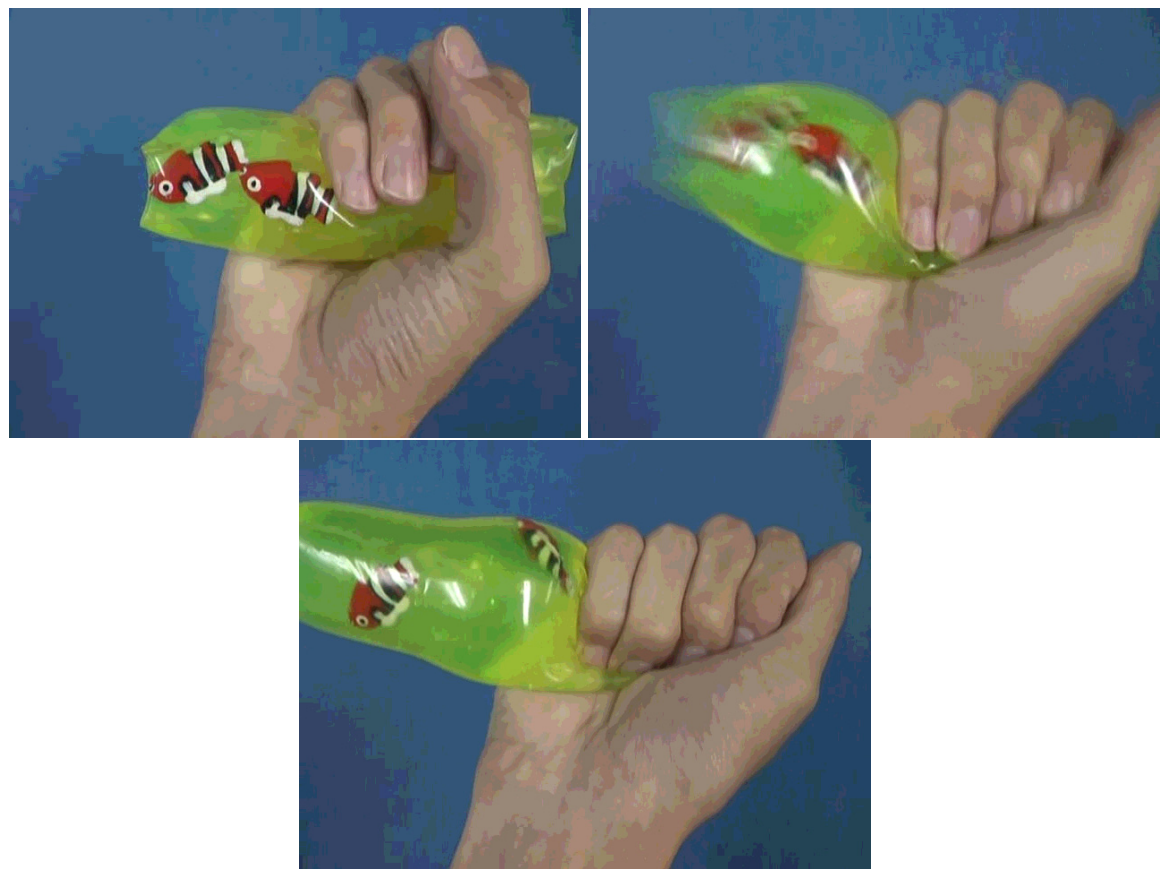

Figure 18. A toy WATER SNAKE, a cylindrical water balloon which will jump out if it grasps tightly 
snake is applied to this principle (Fig.18). The water snake is similar to a cylindrical water balloon. The internal pressure of water inside the balloon is increased by externally grasping and pressing the balloon. Not grasped part is swelled by water pressure and pushed by movement. In the water snake, the external skin rotates and moves inward simultaneously with the outward pushing of the internal skin. Due to this buildup in pressure, no slippage in rotation occurs and the water snake slips from the examiner's grasping hand.

Fig.19 shows the proposed movement principle of the cylindrical soft robot. Magnetic particles inside the water balloon are affected by the external magnetic field and push the inner side of a balloon to the direction of a magnetic field in a manner similar to the examiner's grasping hand, as explained for the case of the toy water snake (Fig.19(2)). This causes the water balloon to rotate (Fig.19(3)(4)).

The key features of the proposed movement principle are

1. The movement does not cause slippage between the contiguous environment and the outside of the cylindrical balloon.

2. The actuator and the battery are not required.

3. The flexible and deformable structure allows the proposed robot to pass through narrow tubes and spaces.

4. The robot can be miniaturized because the structure is simple.

This section also explains the condition that realizes the movement principle. Each parameter is shown in Fig.20. The parameters of the condition are

- $F$ : The force that the external magnetic field imparts to the magnetic particles.

- $a, b$ : The maximum frictional force generated by contact with the contiguous environment.

- $c$ : The force necessary to rotate the flexible skin of the cylindrical balloon and that required to deform it.

The condition required for movement without slippage is given by Eq. 2. This equation determines the value of the external magnetic field to be input.

$$
c<F<a+b
$$

\subsection{Prototype construction for principle verification}

Experimental prototype was constructed an experimental model to verify the proposed principle, as shown in Fig.21. A commercially available toy water snake was used as a cylindrical balloon, which is made of a thermoplastic elastomer. Two types of magnetic particles (TEC-BALL, JFE Techno-Research Corporation)[18], one with specific gravity less than that of a liquid, and the other with specific gravity higher than that of a liquid were placed inside the cylindrical balloon, and the balloon was filled with water. These magnetic particles push the top and bottom parts of the cylindrical balloon simultaneously and generate a well-balanced rotation. The dimensions of the cylindrical balloon became $115 \mathrm{~mm}$ in length, $35 \mathrm{~mm}$ in height, and $42 \mathrm{~mm}$ in width. There is no balloon of dimensions other than this, the size of the abdominal wall model was united with that of the balloon. The black band, which is a material joint, in the center of the cylindrical balloon is used to verify the sliding movement. 


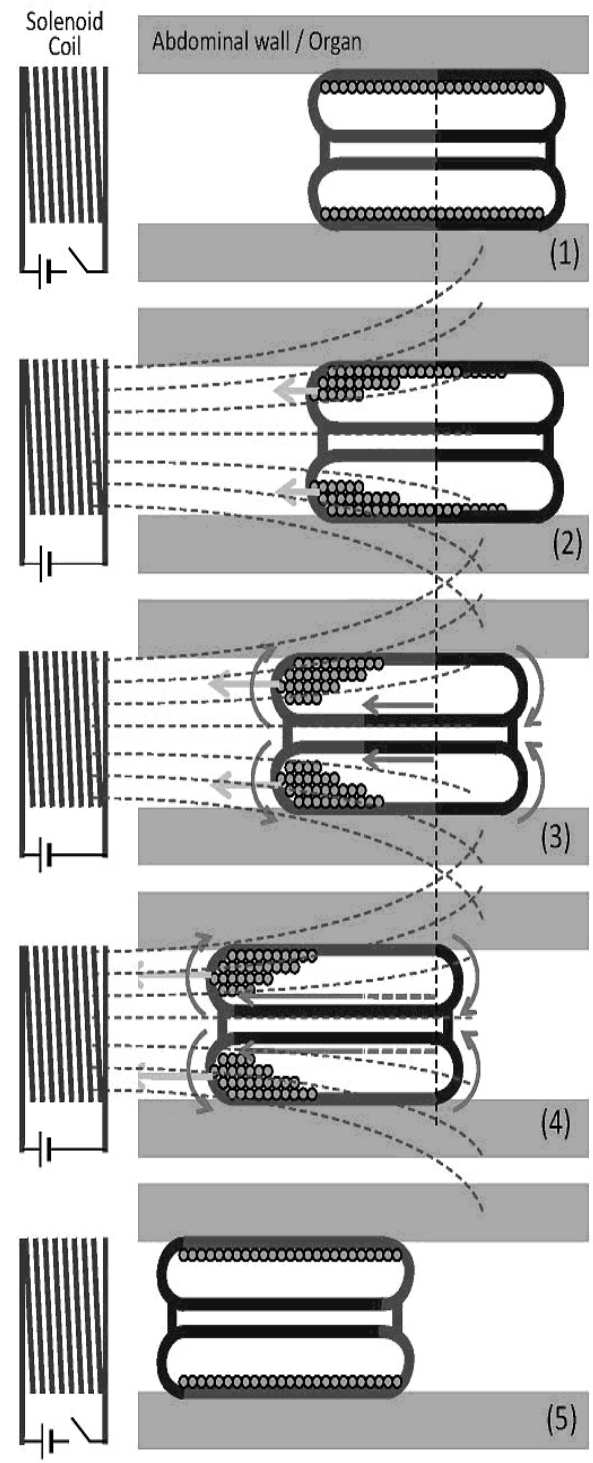

Figure 19. Schematic diagrams of the proposed movement principle of the cylindrical soft robot. Initial state (1), Magnetic particles inside the water balloon are affected by the external magnetic field (2), push the inner side of a balloon, rotate the water balloon to the direction of a magnetic field (3)(4), Magnetic field disappears and rotational movement stops (5). 


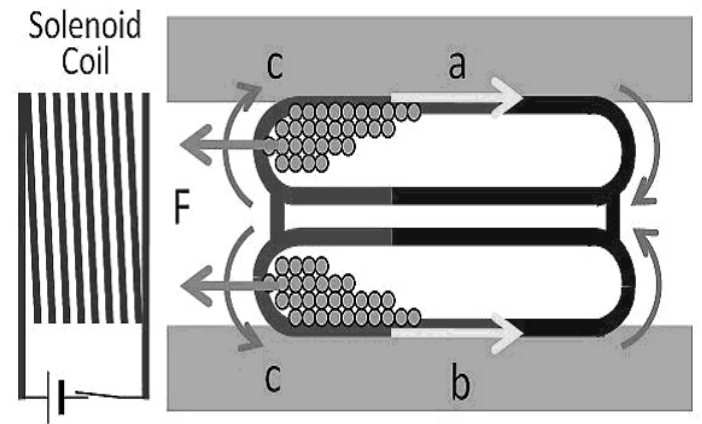

Figure 20. Parameters, $F, a, b$ and $c$ for analyzing the condition that the proposal principle enables the movement.

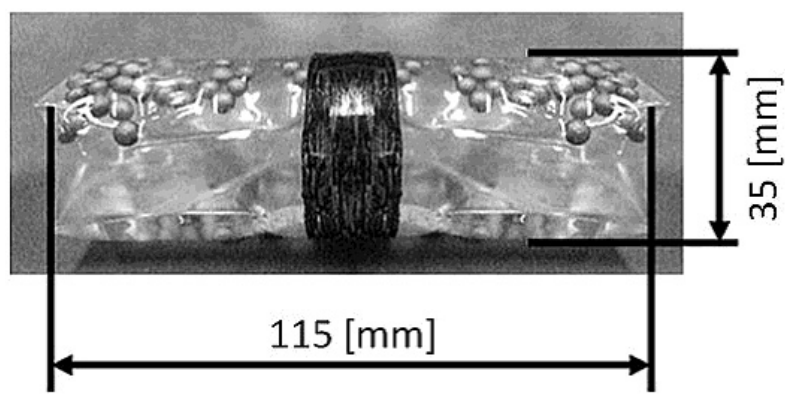

Figure 21. Experimental prototype for the principle verification, it consists of a commercially available toy water snake, two types of magnetic particles and water.

\subsection{Experiment on principle verification}

The sliding movement is measured using an experimental model (Fig.21). The model is pulled with a wire, and it passed between two sheets that simulated internal organs (SEIWA DENTAL Ltd., Japan; Polyvinyl alcohol, $20 \times 10 \times 1 \mathrm{~cm}$ ). The sheet imitates the softness of an abdominal wall but not the colonic peristalsis and the organs' shape. The gap between the two sheets is gradually increased. The force required to slide the model without rotation is measured as frictional force $a, b$, and the force required to rotate and deform the model without slippage is measured as the force necessary for movement $c$ in Eq.(2). The measuring system is shown in Fig.22, it consists of a load cell (KYOWA ELECTRONIC INSTRUMENTS CO., LTD.; LUX-A-1KN), a stage (SIGUMA KOKI CO.,LTD; SGSP 20-30), and a PC. The measured values are shown in Table 1 . The values of the magnetic force necessary for movement were determined at intervals from 28 to $33 \mathrm{~mm}$.

Verification experiment of the proposed movement principle were conducted by use of the experimental prototype(Fig.21), simulated internal organs' sheets and magnetic field generator(Fig.5). 

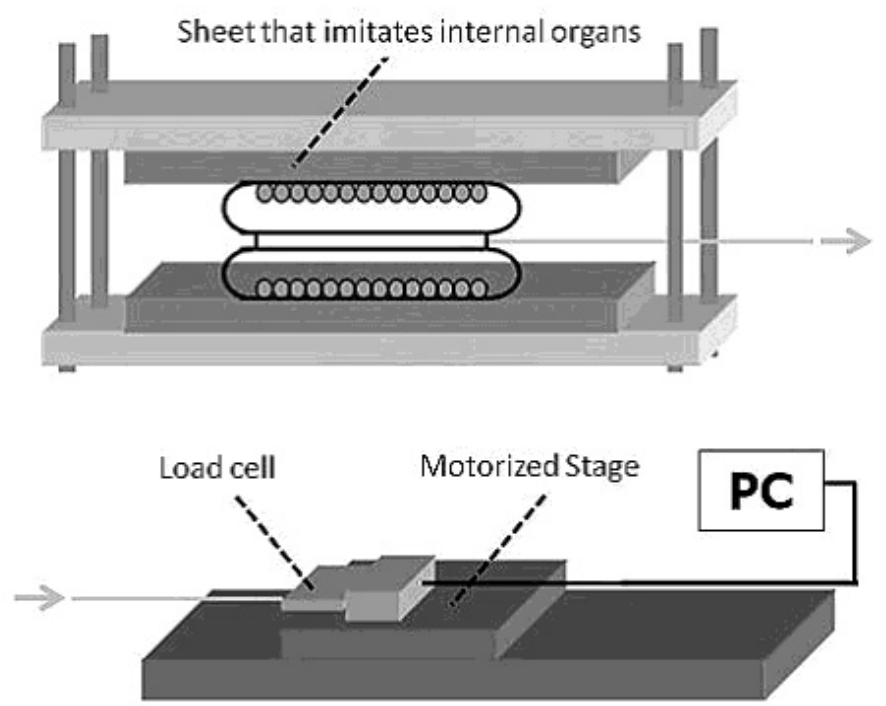

Figure 22. Measuring setup of movement condition. The model is pulled with a wire, and it passed between two sheets that simulated internal organs. The gap between the two sheets is gradually increased.

\begin{tabular}{ccc}
\hline interval[mm] & friction $(\mathrm{a}+\mathrm{b})[\mathrm{N}]$ & deformation $(\mathrm{c})[\mathrm{N}]$ \\
\hline 28 & 2.31 & 0.378 \\
29 & 2.15 & 0.346 \\
30 & 1.99 & 0.328 \\
31 & 1.81 & 0.304 \\
32 & 1.73 & 0.284 \\
33 & 1.69 & 0.263 \\
\hline
\end{tabular}

Table 1. Measured value of movement condition

Fig.23 shows a graph of the measured magnetic force exerted by magnetic particles inside the water ballon. The magnetic force of $0-1.5 \mathrm{~N}$ can be applied to the model by the magnetic field generator.

Eq.(3) shows theoretical formulas of the magnetic force $F$, measured values are consistent with theoretical ones.

$$
\begin{array}{r}
F=m \frac{d H}{d x} \\
m=\chi_{e f f} H V
\end{array}
$$


m: magnetic moment, $H$ :magnetic field,

$x$ : distance from coil, $V$ :volume of material,

$$
\chi_{e f f}: \text { magnetic susceptibility }
$$

In the experiment, the model was positioned at this point and placed between the two sheets that simulate internal organs. The gap between the two sheets was $30 \mathrm{~mm}$. The magnetic force of $0.52 \mathrm{~N}$ was momentarily applied to the model by the magnetic field generator.

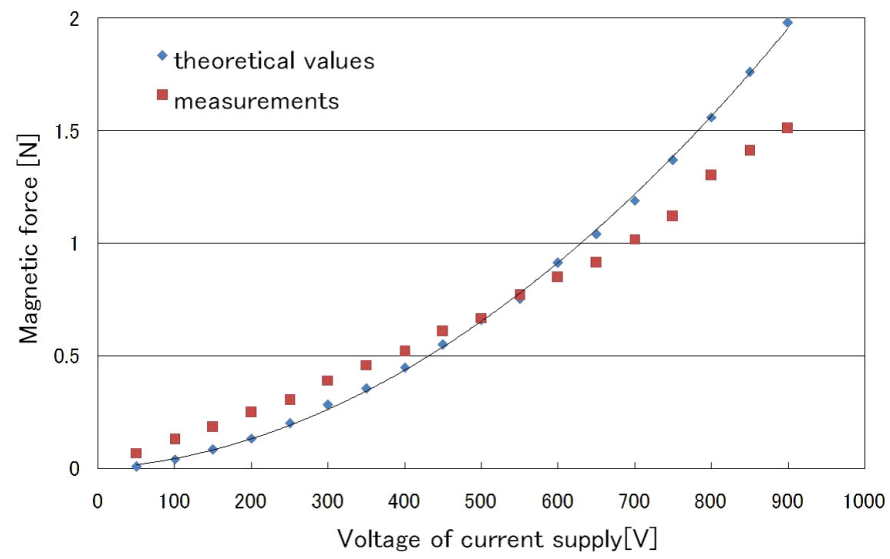

Figure 23. Graph of the measured magnetic force exerted by magnetic particles inside the water balloon.

\subsection{Results and discussions}

The movement of the model is shown in Fig.24. The movement was filmed by a high-speed camera, and the image was captured at intervals of $50 \mathrm{~ms}$. The value written on each figure is the movement distance. The head point on which the triangle is marked moved, while the position of the black band did not move between the two sheets, confirming movement without slippage. The seam part of the cylindrical balloon skin stopped moving, and the total moving distance was $6.2 \mathrm{~cm}$. The moving distance increases by reducing the width of the seam; the method of manufacturing the cylindrical balloon will be improved in the future. Periodic expansion and contraction were observed in the entire film. To improve the efficiency of movement, appropriate material of the cylindrical balloon and sufficient amount of water inside the balloon should be used.

The method of applying the magnetic field to the tube and the patient's body is described. A method using a midair solenoid, as shown in Fig.25, is examined here. The tube or the specimen material is placed in the solenoid, and the part to which the magnetic field is applied is set on the edge of the coil. The magnetic gradient on the edge of the coil generates the magnetic field necessary for movement. 

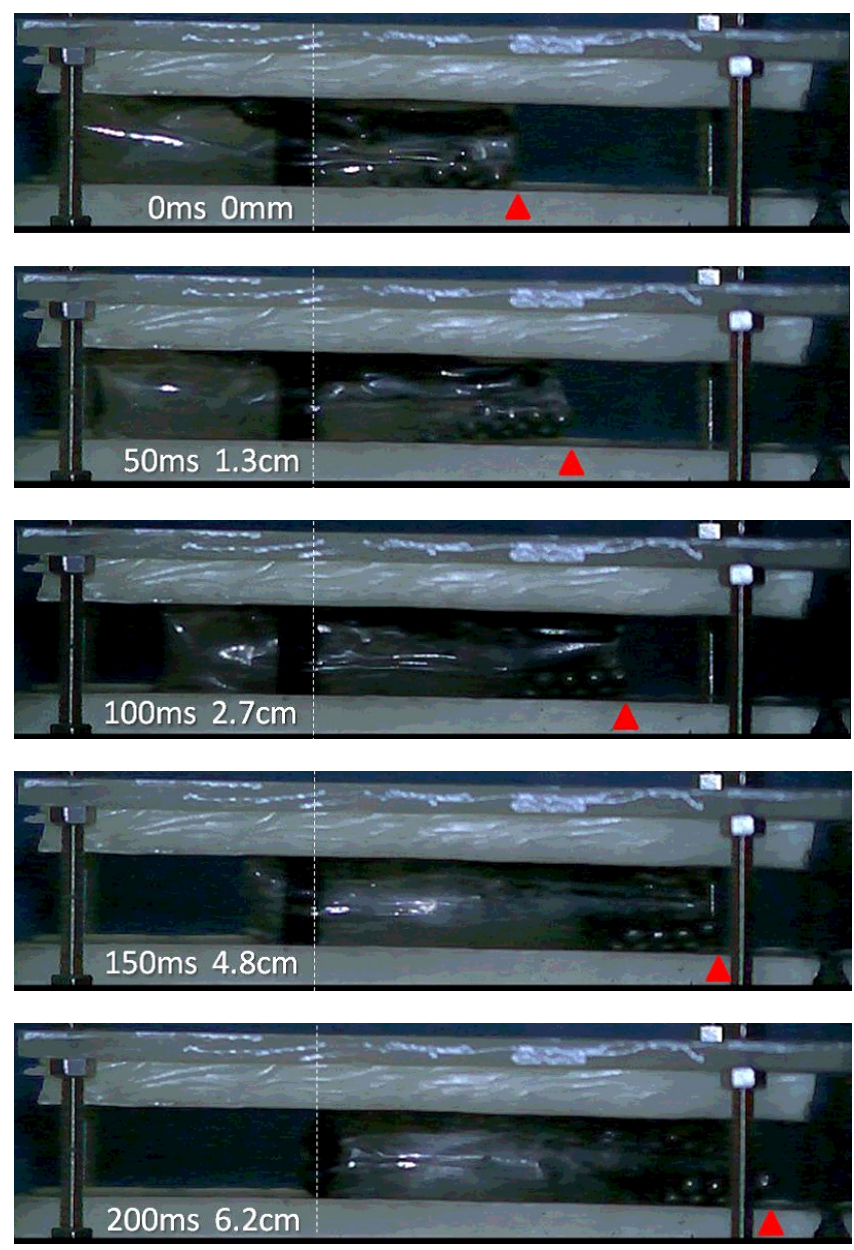

Figure 24. Movement of the model filmed by a high-speed camera, and the image was captured at intervals of $50 \mathrm{~ms}$. The total moving distance was $6.2 \mathrm{~cm}$. 


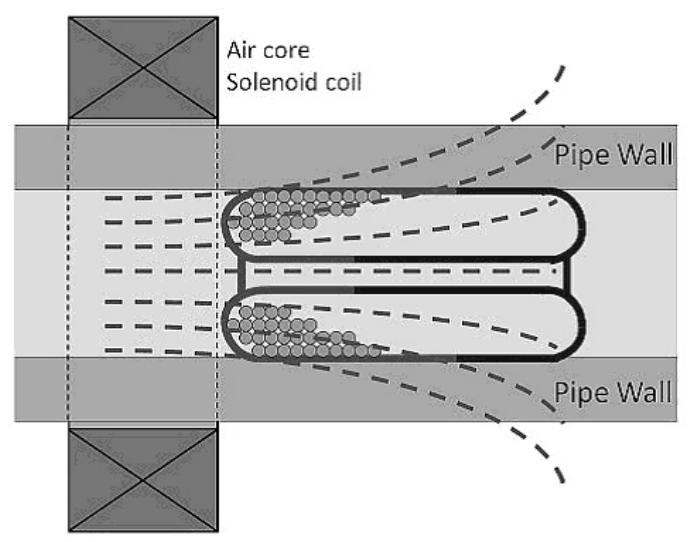

Figure 25. Method of applying the magnetic field to the tube and the patient's body using a midair solenoid.

\section{Conclusions}

This chapter presents a new type of magnetic fluid, a rotational drive and an advanced locomotion method with simple magnetic field.

First, a new type of magnetic fluid and a rotational drive using a simple magnetic field by use of magnetic fluid were presented. It is clear that our proposed driving motions are created by collision of the magnetic particles with the outer cover and the flow resistance between the particles and the cover. The drive for the rolling model was achieved from a small-scale external magnetic field generator and a simple magnetic field control. The device in this study could be applied to new mechanical elements and micro internal rolling objects as a useful medical tool.

Second, an advanced locomotion method that produced non-slipping motion in soft and closed environment by use of magnetic fluid were presented. New movement principle of the robot, which had a soft and deformable body that could move through a confined space was proposed. The mechanism of a toy water snake was applied to this principle. Magnetic particles inside the water balloon were affected by the external magnetic field and push the inner side of a balloon to the direction of a magnetic field. Experimental prototype to verify the proposed principle were conducted, the sliding movement was measured using the model. Confirmatory experiments of movement were conducted in the two sheets that imitated internal organs, the total moving distance was $6.2 \mathrm{~cm}$. Drive a rolling model by simple magnetic field control were succeeded.

\section{Author details}

Makoto Nokata

Department of Robotics, Ritsumeikan University, Japan

\section{References}

[1] http://www.sigma-hc.co.jp/english/index.html 
[2] N. Takesue, H. Asaoka, J. Lin, M. Sakaguchi, G. Zhang and J. Furusho, Development and Experiments of Actuator Using MR Fluid, Proceedings of 2000 IEEE International Conference on Industrial Electronics, Control and Instrumentation, pp.1838-1843, 2000.

[3] T. Kiyota, N. Sugimoto and M. Someya, 3D-Free Rescue Robot System, Proceedings of the 2006 IEEE International Conference on Robotics and Automation (ICRA'06), pp.3983-3988, 2006.

[4] K. Berk Yesin, Philipp Exner, Karl Vollmers and Bradley J. Nelson, Design and Control of In-Vivo Magnetic Microrobots, Proc. of Medical Image Computing and Computer-Assisted Intervention (MICCAI2005), pp.819-826, 2005.

[5] Mathieu, Jean-Baptiste Martel, Sylvain, Magnetic Steering of Iron Oxide Microparticles Using Propulsion Gradient Coils in MRI, 28th Annual International Conference of the IEEE Engineering in Medicine and Biology Society (EMBS'06), pp.472-475, 2006.

[6] CHIBA ATSUSHI, SENDO MASAHIKO, ISHIYAMA KAZUSHI, ARAI KEN'ICHI, Moving of a Magnetic Actuator for a Capsule Endoscope in the Intestine of a Pig, Journal of Magnetics Society of Japan, Vol.29, No.3 pp. 343-346, 2005.

[7] N.TAKESUE, J.FURUSHO, M.SAKAGUCHI, Improvement of Response Properties of MR-Fluid Actuator by Torque Feedback Control, Proceedings of the 2001 IEEE International Conference on Robotics \& Automation, pp.3825-3830, 2001.

[8] J.Furusho and T.Kikuchi, A 3-D rehabilitation System for Upper limbs EMUL and a 6-DOF Rehabilitation System Robotherapist and Other Rehabilitation System with High Safety, Rehabilitation Robotics, I-Tech Education and Publishing, pp.115-136, 2007.

[9] Nokata,M. Kitamura,S. Nakagi,T. Inubushi,T. Morikawa,S., Capsule type medical robot with magnetic drive in abdominal cavity, Proc. of Biomedical Robotics and Biomechatronics, 2008 (BioRob 2008). 2nd IEEE RAS \& EMBS International Conference, pp. 348-353, 2008.

[10] CapsuleEndoscopy, Given Imaging Ltd., http : / /www . capsuleendoscopy . org /

[11] K.Ishiyama, M.Sendoha, A.Yamazakia, K.I Arai: Swimming micro-machine driven by magnetic torque, Sensors and Actuators A: Physical, Vol. 91, Issues 1-2, pp.141-144, 2001.

[12] A.Chiba, M.Sendoh, K.Ishiyama, K.Arai: Magnertic Actuator for capusle endoscope navigation system INTERMAG Asia 2005: Digest of the IEEE International Magnetics Conference.

[13] M. Shikanai et al.: Development of robotic endoscope that locomotes in the colon with flexible helical fin, The 31st Annual International Conference of the IEEE Engineering in Medicine and Biology Society (EMBC2009).

[14] P. Glass, E. Cheung, and M. Sitti: A legged Anchoring Mechanism for Capsule Endoscopes Using Micropatterned Adhesives, IEEE Transactions on Biomedical Engineering, Vol.55, No.12, pp. 2759-2767, 2008.

[15] Eugene Cheung, Mustafa Emre Karagozler, Sukho Park, Byungkyu Kim, and Metin Sitti: A New Endoscopic Microcapsule Robot using Beetle Inspired Microfibrillar Adhesives, Proceedings of the 2005 IEEE/ASME International Conference on Advanced Intelligent Mechatronics, pp.551-557,2005.

[16] M.Nokata et al.: Capsule Type Medical Robot with Magnetic Drive in Abdominal Cavity, IEEE RAS / EMBS International Conference on Biomedical Robotics and Biomechatronics (BioRob 2008), pp.348-353, 2008.

[17] M.Nokata et al.: New magnetic rotational drive by use of magnetic particles with specific gravity smaller than a liquid, 2010 IEEE International Conference on Robotics and Automation (ICRA2010).

[18] T.Fukuda: Characteristic and application development of TECBALL, KINZOKU, vol.79, No.11, 2009 (in Japanese). 